\title{
Predictores de la percepción materna del peso del hijo con sobrepeso-obesidad*
}

\section{Predictors of Maternal Perception about Weight of Son with Overweight-Obesity}

Recibido: agosto 12 de 2012 | Revisado: febrero 3 de 2013 | Aceptado: junio 11 de 2013

\author{
YOLANDA FLORES-PEÑA ** \\ Universidad Autónoma de Nuevo León, México \\ Perla María Trejo-Ortiz *** \\ Universidad Autónoma de Zacatecas, México \\ HERMELINDA ÁVILA-AlPIREZ ***** \\ Universidad AutónOMA DE TAMaulipas, MÉXiCO \\ Velia MARgarita CÁRdenas-VILlaRREAL ****** \\ Universidad Autónoma de Nuevo León, México \\ Alicia UGARTE-ESQUIVEL ******* \\ Universidad Autónoma de Coahuila, México \\ JOSEFINA GALLEGOS-MARTÍNEZ ********** \\ Universidad Autónoma de San Luis Potosí, México \\ Ricardo Martín Cerda-Flores ********* \\ Universidad Autónoma de Nuevo León, México
}

doi:10.11144/Javeriana.UPSY13-2.ppmp

Para citar este artículo: Flores-Peña, Y., TrejoOrtiz, P. M., Ávila-Alpirex, H., Cárdenas-Villarreal, V. M., Ugarte-Esquivel, A., Gallegos-Martínez, J. \& Cerda-Flores, R. M. (2014). Predictores de la percepción materna del peso del hijo con sobrepeso-obesidad. Universitas Psychologica, 13(2). doi:10.11144/Javeriana.UPSY13-2.ppmp

Artículo de investigación. Agradecimientos al Programa de Mejoramiento del Profesorado (PROMEP/103.5/07/2522) por el financiamiento otorgado para la realización del presente proyecto. Los autores están de acuerdo en el orden de autoría y manifiestan que contribuyeron sustancialmente en la elaboración del manuscrito.

** Profesor de Tiempo Completo, Facultad de Enfermería.yflores_mx@yahoo.com.mx

***: Profesor de Tiempo Completo, Área de Ciencias de la Salud.perlu11@yahoo.com.mx

***** Profesor de Tiempo Completo. Unidad Académica Multidisciplinaria Matamoros. avila_alpirez_hermelinda@hotmail.com

******* Profesor de Tiempo Completo. Facultad de Enfermería.velia_margaritac@hotmail.com

**********; Profesor de Tiempo Completo. Escuela de Licenciatura en Enfermería Unidad Torreón. florencia.italia@hotmail.com

*********** Profesor de Tiempo Completo. Facultad de Enfermería.joga2002@hotmail.com

************* Profesor de Tiempo Completo. Facultad de En fermería.ricardocerda_mx@yahoo.com.mx

\section{RES U MEN}

Se identificaron predictores de la percepción materna del peso del hijo (PMPH) con sobrepeso-obesidad (SP-OB). Participaron 2.874 madres e hijos, residentes en el Noreste de México; 951 tenían un hijo con SP-OB. La PMPH se evaluó por palabras (PP) y por imágenes (PI). Por PP, 63.71\% y $74.9 \%$ de madres de hijos con SP-OB no percibieron adecuadamente el peso del hijo y por PI, $27 \%$ y $10.99 \%$. Mediante regresión logística, el modelo para PP fue $\chi^{2}=152.05, g l=9, p=0.001$, varianza explicada $21.5 \%$ y para PI fue $\chi^{2}=192.35, g l=9, p=0.001$, varianza explicada $31.9 \%$. Edad del hijo y si el médico informa de SP en el niño/a, fueron variables que contribuyeron a los modelos. Se concluye que las madres de niños con SP-OB no perciben de forma adecuada esta situación. Se resalta la importancia de informar respecto al SP-OB del hijo.

Palabras clave autores

Niño, obesidad, percepción, percepción del peso, reconocimiento, sobrepeso.

Palabras clave descriptors

\section{A B S T R A C T}

Several predictors of a mother's perception of her child's weight (MPCW) when the child is overweigh-obese (OW-OB) were identified. Participants: 2,842 mothers and children who reside in Mexico's northeastern region, 951 mothers had an OW-OB child. The MPCW was evaluated for words (PW) and images (PI). PW, 63.71\% and $74.9 \%$ of the mothers with an OW-OB child, did not perceive the child's weight adequately and PI, 27\% and $10.99 \%$. Through Logistic Regression Analysis, a model for PW was $\chi^{2}=152.05, g l=9, p=0.001,21.5 \%$ explained variance; and for PI $\chi^{2}$ $=192.35, g l=9, p=0.001,31.9 \%$ explained variance. The child's age, a doctor's report that the child had $\mathrm{OW}$, were some variables that contributed to the models. We concluded that mothers with OW-OB children 
Yolanda Flores-Peña, Perla María Trejo-Ortiz, Hermelinda Ávila-Alpirez, Velia Margarita CárdenasVillarreal, Alicia Ugarte-Esquivel, Josefina Gallegos-Martínez, Ricardo Martín Cerda-Flores

do not adequately perceive this situation. We emphasize the importance of reporting of a child's OW-OB condition. Keywords authors

Child, obesity, overweight, perception, recognition, weight perception

Keywords plus

La obesidad (OB) en la población infantil se ha incrementado. En su etiología interactúan múltiples factores entre los que se encuentran la predisposición genética, el incremento en el consumo de energía, la disminución de la actividad física, el estatus socioeconómico, así como factores socioculturales y las creencias (Gupta, Goel, Shah \& Mirsa, 2012). Un estudio realizado en Italia documentó que la prevalencia alta de $\mathrm{OB}$ infantil se asoció con la no adecuada percepción materna del peso del hijo (PMPH) (Binkin, Spinelli, Baglio \& Lamberti, 2011).

La percepción es el proceso de organizar e interpretar información sensorial para darle significado. La percepción de personas es una de las áreas que constituye la percepción social que se dedica a investigar los procesos y mecanismos por los cuales las personas elaboran y generan la información sobre los otros y sobre sí mismos (Gómez \& Canto, 2000).

Respecto a la PMPH los estudios han reportado que la mayoría de las madres de niños con SP-OB no percibe de forma adecuada el peso de su hijo, tiende a considerarlo con un peso menor. Los estudios se han realizado en los Estados Unidos de Norte América (Doolen, Alpert \& Miller, 2009; Eckstein et al., 2006; Tschamler, Conn, Cook \& Halterman, 2010), Reino Unido (Carnell, Edwards, Croker, Boniface \& Wardle, 2005), Australia (Campbell, Williams, Hampton \& Wake, 2006), Italia (Genovesi et al., 2005), Argentina (Hirschler, Calcagno, Clemente, Aranda \& González, 2008) y Alemania (Warschburger \& Kröller, 2009).

Por otra parte, entre los predictores de la PMPH que se han documentado se encuentran: escolaridad e índice de masa corporal (IMC) materno, edad, IMC y sexo del hijo (Binkin et al., 2011; Genovesi et al., 2005), así como el ingreso económico (Jain et al., 2001).
La percepción de personas puede considerarse como un sistema en el cual la entrada está constituida por tres elementos: a) características de la persona estímulo, en el presente estudio se consideró: edad, sexo e IMC del hijo; b) información almacenada sobre la persona estímulo, para este estudio fueron: que el médico le haya informado a la madre sobre ganancia de peso o presencia de SP en el niño/a $\mathrm{a}^{1 *}$ y la percepción materna de la actividad física que realiza el hijo en comparación a otros niños de la misma edad, c) información sobre el contexto, se consideró: preocupación por el peso del hijo y estado de residencia y d) características del perceptor que para fines del presente estudio fueron: IMC y escolaridad materna e ingreso económico.

Dado que la evidencia indica que del $32.1 \%$ al $87.5 \%$ de las madres de niños con SP-OB no lo perciben (Binkin et al., 2011; Doolen, Alpert \& Miller, 2009; Eckstein et al., 2006; Tschamler et al., 2010; Carnell et al., 2005; Campbell et al., 2006; Genovesi et al., 2005; Hirschler et al., 2008; Warschburger \& Kröller, 2011), además de que los niños con SP cuyas madres no perciben esta situación tienen 4.5 veces más probabilidad de continuar con problemas de exceso de peso en la vida adulta, comparados con aquellos en los que la madre sí lo percibe (Hirschler, Gonzalez, Talgham \& Jadzinsky, 2006), un primer paso en el tratamiento del SP-OB es ayudar a la madre a reconocer esta situación para que pueda tener la disponibilidad de implementar cambios en los hábitos y conductas de los hijos (Eckstein et al., 2006).

Sin embargo, la evidencia disponible respecto a la PMPH en madres mexicanas es escasa (FloresPeña, Trejo-Ortiz, Gallegos-Cabriales \& CerdaFlores, 2009; Lara-García, Flores-Peña, AlatorreEsquivel, Sosa-Briones \& Cerda-Flores, 2011), por lo que iniciamos el estudio de la PMPH en el noreste de México con la participación de diadas, madres y sus hijos de 2 a 17 años inscritos en instituciones educativas públicas (pre-escolar, primaria, secundaria y preparatoria), con el objetivo de identificar

1 * Con el objeto de agilizar la lectura, se opta en adelante por el uso generalizado del término masculino que da por supuesta la inclusión de ambos géneros. 
los predictores de la PMPH con SP-OB por palabras (PP) y por imágenes (PI) en madres de hijos de 2 a 17 años de edad residentes en cinco Estados del noreste de México (Coahuila, Nuevo León, San Luis Potosí, Tamaulipas y Zacatecas).

\section{Método}

\section{Diseño}

Estudio predictivo correlacional (Burns \& Grove, 2009). Se evalúo la PMPH por PP y PI, la cual se clasificó en adecuada y no adecuada. Para identificar los predictores de la PMPH se construyeron dos modelos de regresión logística, considerando como variable dependiente la PMPH por PP y por PI y como variables independientes: 1) edad, 2) sexo, 3) IMC del hijo, 4) el que el médico haya informado a la madre sobre ganancia de peso o presencia de SP en el niño, 5) la percepción materna de la actividad física que realiza el hijo en comparación a otros niños de la misma edad, 6) preocupación por el peso del hijo, 7) estado de residencia, 8) IMC materno, 9) escolaridad y 10) ingreso económico.

\section{Población, muestreo y muestra}

La población de estudio se integró por estudiantes de instituciones de educación pública (2 pre-escolar, 2 primarias, 2 secundarias y 2 preparatorias) en cinco Estados del noreste de México (Coahuila, Nuevo León, Tamaulipas, San Luis Potosí y Zacatecas), un total de 40 instituciones educativas en las cuales se encontraban inscritos 12.295 estudiantes.

Mediante procedimientos estadísticos, se determinó el tamaño de muestra para cada uno de los 5 Estados participantes. Se utilizó el paquete n'Query Advisor ${ }^{\circledR}$ Versión 4.0, para un modelo de regresión logística, prueba unilateral con un nivel de confianza de 0.05 , potencia de prueba de $90 \%$ y Odds Ratio de 2 (Eckstein et al., 2006), lo que resultó en una muestra de 418 hijos (209 niños y 209 niñas) y sus madres, para cada uno de los cinco Estados, se consideró una tasa de no respuesta del 5\%, lo que representó un total de 2.195 diadas.
Al final de la colecta de datos participaron 2.842 diadas madre-hijo y posteriormente de acuerdo al objetivo del estudio, se seleccionaron las diadas que contaban con un hijo con SP-OB.

\section{Mediciones}

Las madres contestaron el cuestionario autoaplicable Percepciones sobre la Apariencia Física y Salud, diseñado por Eckstein et al. (2006). El cuestionario indaga información respecto a las percepciones y preocupaciones que tienen los padres y/o cuidadores sobre la salud y el peso del niño, este cuestionario se puede aplicar a padres y/o cuidadores de niños con edades entre 2 y 17 años. Consta de 19 reactivos, divididos en 6 secciones; en el presente estudio se aplicaron las secciones, uno, dos, tres y seis.

La sección uno está integrada por 8 reactivos que indagan datos sociodemográficos del niño como sexo, edad, condiciones que limitan el crecimiento y actividad física del niño, además de la relación de parentesco con el niño y la escolaridad de los padres y/o cuidadores.

La sección dos consta de 5 reactivos que evalúan la percepción de los padres sobre el peso del niño (PP) mediante el cuestionamiento: "Creo que mi niño/a está...", con opciones de respuesta de "bajo/a de peso", "un poquito bajo/a de peso", "más o menos con el peso correcto", "con un poco de SP y con SP”, además del hecho de que el médico le haya informado sobre ganancia de peso o la presencia de SP en el niño y la percepción materna de las habilidades de actividad física del niño en comparación a otros niños de la misma edad.

En la sección tres, se evalúa la preocupación por el peso de niño y en la sección seis, se presenta una serie de siluetas (PI) de acuerdo al sexo del niño por rangos de edad: 2 a 5 años, 6 a 9 años, 10 a 13 años y 14 a 17 años (Eckstein et al., 2006).

\section{Procedimientos}

Los participantes se reclutaron de escuelas públicas seleccionadas al azar: 2 de nivel pre-escolar, 2 de educación básica y 2 de bachillerato, ubicadas en el área metropolitana de cada uno de los 5 Estados 
Yolanda Flores-Peña, Perla María Trejo-Ortiz, Hermelinda Ávila-Alpirez, Velia Margarita CárdenasVillarreal, Alicia Ugarte-Esquivel, Josefina Gallegos-Martínez, Ricardo Martín Cerda-Flores

participantes. Se realizaron los trámites administrativos para el acceso a las instituciones y, posteriormente, se solicitaron los listados de los alumnos inscritos, a partir de los cuales se seleccionaron los participantes de forma aleatoria y proporcional al tipo de escuela, grado escolar y sexo, mediante el paquete Microsoft ${ }^{\circledR}$ Excel.

A continuación, se solicitó a los directivos, información de la madre a quien se le invitó a una reunión que se realizó en la institución correspondiente, en la cual se expusieron los objetivos de la investigación y los procedimientos que se iban a realizar. Las madres firmaron el consentimiento informado y dieron su autorización para realizar la medición de peso y talla a su hijo. Cuando la madre no asistió a la reunión programada, se seleccionó el niño de la lista después del no localizado. Por otra parte, dado que el IMC materno es un predictor de la $\mathrm{PMPH}$, las mujeres con un embarazo visible fueron sustituidas mediante el proceso antes descrito.

Se aseguró a los participantes la confidencialidad de la información y el anonimato, las madres procedieron a contestar el cuestionario y se realizaron las mediciones de peso y talla a madre e hijo. El peso se midió en kilogramos con la báscula digital marca SECA $813 \circledR$ con capacidad para 150 kilogramos, precisión de $0.1 \mathrm{~kg}$; la talla se midió con un estadímetro marca SECA $214 \AA$, la estatura se registró en el punto más próximo a $0.1 \mathrm{~cm}$.

Posteriormente, se calculó el IMC (peso/talla ${ }^{2}$ ) y el resultado se clasificó en percentiles de acuerdo a edad y sexo del niño, según lo propuesto por la OMS (2012) en: Desnutrición (percentil <3), Bajo peso $(>3 \mathrm{y}<15)$, Peso normal $(>15 \mathrm{y}<85)$, SP $(>85$ pero $<97)$ y OB $(>97)$. Respecto al IMC materno, este también se clasificó de acuerdo a lo establecido por la OMS (2010) en: Bajo peso (< 18.5), peso normal (18.5 a 24.9), pre-obesidad (25 a 29.9), OB I (30 a 34.9), OB II (35 а 39.9) y OB III $(>40)$.

El estudio fue aprobado por el Comité Investigación y Ética de la Facultad de Enfermería de la Universidad Autónoma de Nuevo León y se ajustó a las Normas Éticas de la Declaración de Helsinki de 1973.

\section{Análisis estadístico}

Los datos se capturaron y analizaron en el paquete estadístico Statistical Package for the Social Sciences (SPSS) versión 20 para Windows. Se obtuvieron estadísticas descriptivas de las variables sociodemográficas de las diadas participantes. Primero se evalúo la PMPH por PP, se consideró percepción adecuada a) cuando las madres de hijos con SP los describieron como con un poco de SP y b) cuando las madres de niños con $\mathrm{OB}$ los describieron con SP. Respecto a la PI se consideró adecuada cuando las madres de hijos con SP-OB seleccionaron una imagen media o mayor.

Posteriormente, para identificar los predictores de la PMPH se aplicó regresión logística, y mediante el procedimiento atrás (backward) se identificaron las variables que contribuían al modelo; finalmente, se calculó la probabilidad de la percepción adecuada para PP y PI de las variables.

\section{Resultados}

Participaron 2.842 diadas (madre-hijo). Respecto a la categoría de peso del hijo, se identificó bajo peso en el 11.4\% $(n=325)$, peso normal en el $55.1 \%$ ( $n$ $=1.566)$, el SP se presentó en el $15.1 \%(n=441)$ y la OB en el $17.9 \%(n=510)$.

Posteriormente, de acuerdo al objetivo del estudio, se consideraron las diadas que contaban con un hijo con SP-OB. Respecto a la información materna, la edad osciló entre los 19 y 67 años con una media de 37.3 años $(D E=7.1)$, escolaridad promedio de 10.24 años $(D E=3.9)$, valor mínimo de 0 y máximo de 25 años. La mayoría de las madres se dedicaba al hogar $(67.1 \%, n=638)$ y estaban casadas $(80.9 \%, n=769)$. El ingreso económico se ubicó en $\$ 6.856 .94$ pesos mexicanos $(D E=\$ 6.449 .5)$, valor mínimo de $\$ 800$ y máximo de $\$ 65.000$. El $36.2 \%(n=344)$ de las madres tenía Pre-Obesidad y el $45.4 \%(n=431)$ presentó algún grado de $\mathrm{OB}$.

Respecto a los hijos, el 17.4\% $(n=165)$ cursaban educación pre-escolar, 31.2\% $(n=297)$ primaria, el $31.5 \%(n=300)$ secundaria y $19.9 \%(n=189)$ preparatoria. El 49.1\% $(n=467)$ pertenecían al sexo femenino y el $50.9 \%(n=484)$ al masculino. 
La edad promedio del hijo fue de 11 años $(D E=$ 4.1) con un valor mínimo de 2 años y un máximo de 17 años.

$\mathrm{Al}$ evaluar la PMPH por PP, se encontró que la mayoría de las madres de niños con SP no percibieron de forma adecuada el peso de su hijo; lo perciben con peso normal, poco bajo peso y bajo peso $(63.71 \%, n=281)$ y respecto a las madres de niños con OB el 74.9\% $(n=382)$ lo refirió con SP, peso normal, poco bajo peso y bajo peso (Tabla 1).

La Tabla 2 muestra la PMPH por PI; se encontró que el 27\% $(n=119)$ de las madres de niños con SP y el $10.99 \%(n=56)$ de madres de niños con $\mathrm{OB}$, no percibieron de forma adecuada el peso de su hijo (selección de la imagen 1, 2 y 3).

Para identificar los predictores de la PMPH se aplicó regresión logística; la Tabla 3 muestra el modelo significativo para PP $\left(\chi^{2}=152.05, g l=9\right.$, $p<0.001)$ con una varianza explicada de $21.5 \%$. Las variables que contribuyeron al modelo fueron: a) edad del hijo, b) que el médico haya informado a la madre sobre ganancia de peso o presencia de SP en el niño, c) percepción materna de la actividad física que realiza el hijo en comparación con otros niños de la misma edad y d) preocupación por el peso del hijo.

Posteriormente, se calculó la probabilidad del valor ajustado para los predictores que contribuyeron al modelo para la adecuada PMPH por PP, y se encontró que cuando la madre responde afirmativamente que el médico le ha informado sobre ganancia de peso o la presencia de SP en el niño, sí la madre percibe que cuando su hijo corre es usualmente más lento en comparación con otros niños de su misma edad; y cuando señala estar preocupada por el peso del hijo, la probabilidad de que perciba

TABLA 1

Percepción materna del peso del hijo con SP-OB por PP

\begin{tabular}{lcccc}
\hline \multicolumn{1}{c}{ Creo que mi niño(a) esta... } & \multicolumn{2}{c}{ SP } & \multicolumn{2}{c}{ OB } \\
\cline { 2 - 5 } & $n$ & $\%$ & 1 & 0.19 \\
\hline Bajo(a) peso & 4 & 0.9 & 5 & 0.98 \\
Poco Bajo(a) peso & 7 & 1.59 & 95 & 18.63 \\
Peso normal & 270 & 61.22 & 281 & 55.1 \\
Con SP & 142 & 32.2 & 128 & 25.1 \\
Con bastante SP & 18 & 4.09 & 510 & 100 \\
\hline Total & 441 & 100 & \\
\hline
\end{tabular}

$\chi^{2}=210.693, g l=4, p=0.001$.

Fuente: elaboración propia.

\section{TABLA 2}

Percepción materna del peso del hijo con SP-OB por PI

\begin{tabular}{|c|c|c|c|c|}
\hline \multirow[t]{2}{*}{ Imagen } & \multicolumn{2}{|c|}{ SP } & \multicolumn{2}{|c|}{ OB } \\
\hline & $n$ & $\%$ & $n$ & $\%$ \\
\hline 1 & 6 & 1.36 & 4 & 0.79 \\
\hline 2 & 37 & 8.4 & 15 & 2.94 \\
\hline 3 & 76 & 17.24 & 37 & 7.26 \\
\hline 4 & 153 & 34.69 & 76 & 14.9 \\
\hline 5 & 113 & 25.62 & 139 & 27.25 \\
\hline 6 & 50 & 11.33 & 164 & 32.16 \\
\hline 7 & 6 & 1.36 & 75 & 14.7 \\
\hline Total & 441 & 100 & 510 & 100 \\
\hline
\end{tabular}

$\chi^{2}=167.121, g l=6, p=0.001$.

Fuente: elaboración propia. 
Yolanda Flores-Peña, Perla María Trejo-Ortiz, Hermelinda Ávila-Alpirez, Velia Margarita CÁrdenasVillarreal, Alicia Ugarte-Esquivel, Josefina Gallegos-Martínez, Ricardo Martín Cerda-Flores

TABLA 3

Predictores de la percepción materna del peso del hijo con SP-OB por PP

\begin{tabular}{|c|c|c|c|c|c|c|}
\hline Modelo & \multicolumn{2}{|l|}{$\chi^{2}$} & $\mathrm{gl}$ & \multicolumn{2}{|c|}{ Valor de $p$} & $\mathrm{R}^{2}$ \\
\hline Regresión Logística & 152.05 & & 9 & & & 0.215 \\
\hline \multicolumn{7}{|c|}{ Efecto de las variables sobre la percepción materna del peso del hijo (PMPP) } \\
\hline Variable & $\beta$ & ES & Wald & $\mathrm{gl}$ & $\mathrm{p}$ & IC 95\% \\
\hline Edad hijo & 0.251 & 0.028 & 80.793 & 1 & 0.001 & $1.217-1.358$ \\
\hline $\begin{array}{l}\text { El médico le ha informado sobre ganancia de peso o la } \\
\text { presencia de SP en el niño/a }\end{array}$ & & & 9.521 & 2 & 0.009 & \\
\hline No & -0.353 & 0.157 & 5.086 & 1 & 0.024 & $0.517-0.955$ \\
\hline Sí & 0.181 & 0.169 & 1.144 & 1 & 0.285 & $0.860-1.669$ \\
\hline No sé & 0 & & & & & \\
\hline Cuando mi niño/a corre es usualmente... & & & 24.591 & 2 & 0.001 & \\
\hline Más rápido que otros niños/as & -0.412 & 0.274 & 2.265 & 1 & 0.132 & $0.387-1.133$ \\
\hline Tan rápido como otros niños/as & -0.904 & 0.185 & 23.931 & 1 & 0.001 & $0.282-0.582$ \\
\hline Más lento que otros niños/as & 0 & & & & & \\
\hline Estoy preocupada por el peso de mi niño/a & & & 22.445 & 4 & 0.001 & \\
\hline Totalmente de acuerdo & -1.662 & 0.399 & 17.379 & 1 & 0.001 & $0.087-0.415$ \\
\hline De acuerdo & -0.911 & 0.345 & 6.983 & 1 & 0.008 & $0.205-0.79$ \\
\hline Neutral & -0.419 & 0.285 & 2.167 & 1 & 0.141 & $0.377-1.149$ \\
\hline En desacuerdo & -0.354 & 0.181 & 3.846 & 1 & 0.05 & $0.493-1$ \\
\hline Totalmente en desacuerdo & 0 & & & & & \\
\hline Constante & -1.744 & 0.327 & 28.485 & 1 & 0.001 & \\
\hline
\end{tabular}

Fuente: elaboración propia.

TABLA 4

Probabilidad de percibir de forma adecuada el peso del hijo por PP

\begin{tabular}{lcccc}
\hline & $n$ & Media & Mdn & DE \\
\cline { 1 - 2 } $\begin{array}{l}\text { El médico le ha informado sobre ganancia de peso o la presen- } \\
\text { cia de SP en el niño/a }\end{array}$ & & & & \\
\cline { 1 - 3 } No & 685 & 0.23 & 0.21 & 0.14 \\
Sí & 222 & 0.42 & 0.42 & 0.17 \\
No sé & 32 & 0.37 & 032 & 0.19 \\
Total & 939 & 0.28 & 0.26 & 0.17 \\
\hline Cuando mi niño/a corre es usualmente... & & & & \\
Más rápido que otros niños/as & 117 & 0.25 & 0.25 & 0.14 \\
Tan rápido como otros niños/as & 605 & 0.22 & 0.21 & 0.13 \\
Más lento que otros niños/as & 217 & 0.45 & 0.47 & 0.17 \\
Total & 939 & 0.28 & 0.26 & 0.17 \\
\hline Estoy preocupada por el peso de mi niño/a & & & & \\
Totalmente desacuerdo & 95 & 0.08 & 0.07 & 0.06 \\
En desacuerdo & 87 & 0.14 & .13 & 0.1 \\
Neutral & 96 & 0.23 & 0.25 & 0.12 \\
De acuerdo & 321 & 0.28 & 0.26 & 0.15 \\
Totalmente desacuerdo & 340 & 0.37 & 0.34 & 0.17 \\
Total & 939 & 0.28 & 0.26 & 0.17 \\
\hline
\end{tabular}

Fuente: elaboración propia. 
de forma adecuada el SP-OB de su hijo, es mayor. Las estadísticas descriptivas para los valores de probabilidad se muestran en la Tabla 4.

La Tabla 5 presenta el modelo de regresión logística con las variables que contribuyen a la $\mathrm{PMPH}$ por PI; se identificó un modelo significativo $\left(\chi^{2}=\right.$ 192.353, $g l=9, p<0.001)$, varianza explicada del $31.9 \%$. Las variables que contribuyeron al modelo fueron: a) IMC del hijo, b) que el médico le haya informado a la madre sobre ganancia de peso o presencia de SP en el niño, c) percepción materna de la actividad física que realiza el hijo en comparación a otros niños de la misma edad y d) el ingreso económico mensual.

Finalmente, se calculó la probabilidad del valor ajustado para los predictores que contribuyeron al modelo para la adecuada PMPH por PI; se encontró que cuando la madre responde afirmativamente que el médico le ha informado sobre ganancia de peso o la presencia de SP en el niño y cuando la madre

TABLA 5

Predictores de la percepción materna del peso del hijo con SP-OB por PI

\begin{tabular}{|c|c|c|c|c|c|c|}
\hline Regresión Logística & $\begin{array}{c}\chi^{2} \\
192.353\end{array}$ & & $\begin{array}{l}\text { gl } \\
9\end{array}$ & & $\begin{array}{r}\text { de } p \\
0\end{array}$ & $\begin{array}{c}\mathbf{R}^{2} \\
0.319\end{array}$ \\
\hline \multicolumn{7}{|c|}{ Efecto de las variables sobre la percepción materna del peso del hijo (PMPI) } \\
\hline Variable & $\beta$ & ES & Wald & gl & $\mathrm{p}$ & IC 95\% \\
\hline IMC hijo & 0.251 & 0.028 & 80.793 & 1 & 0.001 & $1.217-1.358$ \\
\hline $\begin{array}{l}\text { El médico le ha informado sobre ganancia de peso o la } \\
\text { presencia de SP en el niño/a }\end{array}$ & & & 15.428 & 2 & 0.001 & \\
\hline No & -0.71 & 0.252 & 8.008 & 1 & 0.005 & $0.299-0.803$ \\
\hline Sí & 0.89 & 0.337 & 7.126 & 1 & 0.008 & $1.27-4.762$ \\
\hline No sé & 0 & & & & & \\
\hline Cuando mi niño/a corre es usualmente & & & 10.441 & 2 & 0.005 & \\
\hline Más rápido que otros niños/as & -1.271 & 0.394 & 10.425 & 1 & 0.001 & $0.13-0.607$ \\
\hline Tan rápido como otros niños/as & -0.85 & 0.337 & 6.365 & 1 & 0.012 & $0.221-0.827$ \\
\hline Más lento que otros niños/as & 0 & & & & & \\
\hline Ingreso económico mensual & -0.000049 & 0 & 11.677 & 1 & 0.001 & $1-1$ \\
\hline Constante & -2.6346 & 0.73 & 13.013 & 1 & 0.001 & \\
\hline
\end{tabular}

Fuente: elaboración propia.

TABLA 6

Probabilidad de percibir de forma adecuada el peso del hijo por PI

\begin{tabular}{|c|c|c|c|c|}
\hline & $n$ & Media & $M d n$ & $\mathrm{DE}$ \\
\hline \multicolumn{5}{|l|}{$\begin{array}{l}\text { El médico le ha informado sobre ganancia de peso o la presencia de SP en el } \\
\text { niño/a }\end{array}$} \\
\hline No & 648 & 0.76 & 0.82 & 0.18 \\
\hline $\mathrm{Si}$ & 205 & 0.96 & 0.98 & 0.04 \\
\hline No se & 29 & 0.86 & 0.92 & 0.15 \\
\hline Total & 882 & 0.81 & 0.88 & 0.18 \\
\hline \multicolumn{5}{|l|}{ Cuando mi niño/a corre es usualmente... } \\
\hline Más rápido que otros niños/as & 112 & 0.68 & 0.71 & 0.22 \\
\hline Tan rápido como otros niños/as & 565 & 0.79 & 0.84 & 0.17 \\
\hline Más lento que otros niños/as & 205 & 0.94 & 0.96 & 0.07 \\
\hline Total & 882 & 0.81 & 0.88 & 0.18 \\
\hline
\end{tabular}

Fuente: elaboración propia. 
Yolanda Flores-Peña, Perla María Trejo-Ortiz, Hermelinda Ávila-Alpirez, Velia Margarita CárdenasVillarreal, Alicia Ugarte-Esquivel, Josefina Gallegos-Martínez, Ricardo Martín Cerda-Flores

señaló que cuando su hijo corre es usualmente más lento en comparación con otros niños de su misma edad, se incrementa la probabilidad de que perciba de forma adecuada el SP-OB del hijo.

\section{Discusión}

La percepción de una persona implica organizar información sobre el sujeto y atribuirle propiedades que culminan en un juicio de carácter evaluativo, formado a partir de características físicas observables, así como de características y estados no observables del sujeto percibido. Es un proceso que va más allá de la apariencia física, en el cual intervienen mecanismos emocionales, cognoscitivos y motivacionales, entre otros, que afectan la información, por lo que el juicio que se emite no siempre corresponde a la entrada sensorial (Gómez \& Canto, 1997).

De la misma forma en el presente estudio se encontró que la mayoría de las madres participantes de hijos con SP-OB no percibieron de forma adecuada esta situación, consideran como inferior el peso de su hijo; hallazgo que también se ha documentado en países como Estados Unidos de Norte América (Doolen et al., 2009; Eckstein et al., 2006; Tschamler et al., 2010), Italia (Genovesi et al., 2005), Alemania (Warschburger \& Kröller, 2009) y en madres residentes en el Estado de Nuevo León, México (Flores-Peña et al., 2009).

Otro hallazgo que es de interés resaltar es que cuando se aplica la prueba PP, es decir, cuando se plantea el cuestionamiento: "Creo que mi niño está...”, es aún más alta la proporción de madres con una PMPH no adecuada. Cabe mencionar que la prueba PP tiene menor sensibilidad y especificidad en comparación con la prueba PI (Eckstein et al., 2006; Flores-Peña et al., 2009), sin embargo, es importante considerar que al aplicar la prueba PP no se realiza ninguna intervención y que es esta la percepción que antecede las acciones y dificultades que la madre experimenta para controlar o no el peso del hijo. Por otra parte, un estudio realizado en Italia documentó que la alta prevalencia de $\mathrm{OB}$ infantil se asoció con la $\mathrm{PMPH}$ no adecuada, lo que sugiere que dado que la $\mathrm{OB}$ infantil es común, esta se percibe como normal (Binkin et al., 2011).

De la misma forma, reflexionando respecto al hallazgo anterior, surge la interrogante de si existe la posibilidad de atribuirlo a la creencia dominante de que el SP-OB son sinónimos de un niño sano, además de que el SP-OB del hijo no representa preocupación para la madre, mientras esta situación no interfiera con las actividades diarias que el hijo realiza (Jain et al., 2001); de la misma forma, otros autores señalan que existe en las madres una incapacidad para percibir de forma adecuada el SP-OB de su hijo y que podría ser más fácil que perciban de forma adecuada el SP-OB cuando se presenta en niños no emparentados, es decir, aquellos niños con los que no se comparten lazos consanguíneos (Huang et al., 2007; Warschburger \& Kröller, 2009), lo que también se ha documentado en madres residentes en el Estado de Tamaulipas, México (Lara-García et al., 2011).

$\mathrm{El}$ incremento en la prevalencia del SP-OB infantil es una situación en la que intervienen múltiples factores; desde factores genéticos, reducción de la actividad física, incremento en el consumo de calorías y nivel socioeconómico alto, además de factores socioculturales como la sobreprotección y el hecho de que los padres presionen al hijo para alimentarse, así como las creencias tradicionales falsas respecto a la salud y nutrición, arraigadas en madres de niños con SP-OB (Gupta et al., 2012).

Las madres tienen la creencia de que el patrón de crecimiento esta prefijado o predestinado y que es atribuible a la herencia genética, por lo que es prácticamente imposible alterar el peso del niño; se ha señalado que padres de niños con $\mathrm{OB}$ creen que la $\mathrm{OB}$ desaparecerá cuando sus hijos sean mayores (Jain et al., 2001; Hugues, Sherman, \& Whitaker, 2010), sin embargo, se sabe que la mayoría de estos niños seguirán presentando $\mathrm{OB}$ en la vida adulta (Wardle, Brodersen, Cole, Jarvis \& Boniface, 2006).

Respecto a los predictores de la $\mathrm{PMPH}$, el presente estudio identificó que a mayor edad e IMC del hijo, mayor ingreso económico; cuando la madre responde afirmativamente que el médico le ha informado sobre ganancia de peso o la presencia de 
SP en el niño, si la madre señala que cuando su hijo corre es usualmente más lento en comparación con otros niños de su misma edad y cuando la madre se siente preocupada por el peso de su hijo, se incrementa la probabilidad de una adecuada PMPH.

Respecto a la edad del hijo, se identificó que a mayor edad es mayor la probabilidad de una adecuada PMPH, hallazgo similar a lo reportado por Genovesi et al. (2005), quienes refieren que las madres de los niños mayores de seis años, tienen mayor probabilidad de percibir de forma adecuada el peso de su hijo, por otra parte, de acuerdo Jain et al. (2001) que realizaron un estudio cualitativo y documentaron que las madres de niños pequeños consideran que su hijo dejará de ser obeso cuando tenga más edad, empiece a crecer y ser más activo.

Así mismo, se identificó que a mayor IMC del hijo mayor probabilidad de una adecuada PMPH, lo que coincide con lo reportado por Binkin et al. (2011) quienes señala que es más probable que la madre perciba de forma adecuada el peso de su hijo cuando este tiene bastante SP, mientras que un niño con poco SP se percibe como normal. En relación con el ingreso económico, en el presente estudio se identificó que a menor ingreso económico es mayor la probabilidad de una adecuada PMPH; a este respecto se ha documentado una relación entre el incremento en la prevalencia del SP-OB infantil y el nivel socioeconómico alto (Gupta et al., 2012).

Por otra parte, respecto al hallazgo de cuando la madre responde afirmativamente que se le ha informado sobre ganancia de peso o la presencia de SP en el niño, es importante señalar que el presente trabajo identificó que a la mayoría de las madres participantes no se les ha informado respecto a que su hijo presenta SP-OB. A este respecto, es posible señalar que algunos estudios que evalúan los servicios de salud han identificado que a los padres les gustaría tratar con el personal de salud tópicos como desarrollo del niño, patrones de sueño y descanso, así como aspectos del contexto familiar y social (Kuo et al., 2006); otros estudios señalan que la atención en salud que está orientada a la ejecución de procedimientos, tales como mediciones antropométricas de peso y talla, las cuales solo se registran en el expediente del niño sin comunicár- selas a la madre o familiar responsable que acude con el menor a la consulta (Flores-Peña, Ortiz-Félix \& Cárdenas-Villarreal, 2012).

De acuerdo a lo anterior, se recomienda que el personal médico y de la salud en el ámbito clínico o comunitario informe a la madre cuando su hijo presente problemas de peso, así como detectarlos lo más temprano posible y no cuando el niño tenga probablemente años de evolución con los problemas de peso y un riesgo mayor de comorbilidades; así mismo, se recomienda hacer énfasis en las complicaciones presentes y futuras del SP-OB, dado que cuando la madre refirió sentirse preocupada por el peso de su hijo, la probabilidad de una adecuada PMPH fue mayor.

Otro de los predictores de la adecuada PMPH fue el hecho de que la madre perciba a su hijo más lento que otros niños de su misma edad al realizar actividad física, lo que muestra coincidencia con un estudio realizado en Finlandia que reportó una relación inversa entre estas variables, es decir, que si los padres perciben a sus hijos como físicamente activos con menor frecuencia los perciben como con SP (Vanhala, Keinänen-Kiukaanniemi, Kaikkonen, Laitinen \& Korpelainen, 2011). Por tanto, se sugiere promover en el ámbito escolar y/o comunitario actividades que permitan la oportunidad para que la madre compare las habilidades de actividad física de su hijo con las de otros niños.

Otros estudios han identificado que el IMC materno predice la $\mathrm{PMPH}$, aspecto que no se confirma en el presente estudio, sin embargo, dado que casi la mitad de las madres participantes presentaron exceso de peso, sin contar otro porcentaje que presentó preobesidad, este hallazgo señala que los hábitos y costumbres se comparten en el contexto familiar, por lo que se recomienda explorar los hábitos y costumbres de alimentación y actividad física. Por otra parte, dado que se ha identificado que las siluetas tienen mayor sensibilidad y especificidad (Eckstein et al., 2006; Flores-Peña et al., 2009) en comparación al hecho de solo aplicar cuestionamientos respecto al peso del hijo, se recomienda considerarlas como una herramienta para la práctica clínica.

De acuerdo a los hallazgos del presente estudio se concluye que las madres de hijos con SP-OB 
Yolanda Flores-Peña, Perla María Trejo-Ortiz, Hermelinda Ávila-Alpirez, Velia Margarita CárdenasVillarreal, Alicia Ugarte-Esquivel, Josefina Gallegos-Martínez, Ricardo Martín Cerda-Flores

de 2 a 17 años de edad residentes en el noreste de México no perciben de forma adecuada esta situación, subestiman el peso de su hijo, proporción que es mayor cuando solo se realizan cuestionamientos respecto al peso de su hijo. Se identificó que a mayor IMC y edad del hijo, menor ingreso económico; cuando la madre refiere que el médico le ha comentado sobre ganancia de peso o SP en su niño, cuando la madre percibe a su hijo como más lento que otros niños de su misma edad y cuando señala estar preocupada por el peso de su hijo, la probabilidad de una adecuada PMPH se incrementa. Por otra parte, dado que no se identificó el Estado de residencia como predictor de la $\mathrm{PMPH}$, es posible señalar que estos hallazgos pueden ser generalizados a las madres residentes en el noreste de México.

Como ya se mencionó, la PMPH con SP-OB es un factor que puede motivar o no la participación materna en programas de intervención para disminuir y controlar el peso del hijo, por lo que se recomienda desarrollar intervenciones abordando los predictores identificados en el presente estudio. Finalmente, se considera que intervenir en modificar la PMPH con SP-OB es un reto, dado que en la percepción de personas confluyen entre otros mecanismos emocionales y cognitivos, y que en el caso particular de la PMPH, el lazo filial madre-hijo, además del hecho de que tener un hijo sano es sinónimo de éxito para la madre, son situaciones que se constituyen en un reto al momento de diseñar y planear intervenciones efectivas.

\section{Referencias}

Binkin, N., Spinelli, A., Baglio, G. \& Lamberti, A. (2011). What is common becomes normal: The effect of obesity prevalence on maternal perception. Nutrition, Metabolism $\mathcal{E}$ Cardiovascular Diseases. Dec 31. [Epub ahead of print]. http://dx.doi. org/10.1016/j.numecd.2011.09.006

Burns, N. \& Grove, S. K. (2009). The practice of nursing research: Appraisal, synthesis, and generation of evidence (6a. ed.). Missouri. Elsevier.

Campbell, M. W., Williams, J., Hampton, A. \& Wake, M. (2006). Maternal concern and perceptions of overweight in Australian preschool-aged children. Medical Journal of Australia, 184(6), 274-277.

Carnell, S., Edwards, C., Croker, H., Boniface, D. \& Wardle J. (2005). Parental perceptions of overweight in 3-5 y olds. International Journal of Obesity, 29(4), 353-355. http://dx.doi.org/10.1038/ sj.ijo.0802889

Doolen, J., Alpert, P. T. \& Miller, S. K. (2009). Parental disconnect between perceived and actual weight status of children: A metasynthesis of the current research. Journal of the American Academy of Nurse Practitioners, 21(3), 160-166. http://dx.doi. org/10.1111/j.1745-7599.2008.00382.x

Eckstein, K. C., Mikhail, L. M., Ariza, A. J., Thomson, J. S., Millard, S. C. \& Binns, H. J. (2006). Parents' perceptions of their child's weight and health. Pediatrics, 117(3), 681-690. http://dx.doi.org/10.1542/ peds.2005-0910

Flores-Peña, Y., Ortiz-Félix, R. E. \& Cárdenas-Villarreal, V. M. (2012, marzo-abril). Percepciones maternas del cuidado del niño sano. Revista Latino-Americana de Enfermería. Enfermagen [Internet], 20(2), [7 pantallas]. Disponible en file:///C:/Users/HP/ Desktop/48503-58975-1-PB.pdf

Flores-Peña, Y., Trejo-Ortiz, P. M., Gallegos-Cabriales, E. C. \& Cerda-Flores, R. M. (2009). Validez de dos pruebas para evaluar la percepción materna del peso del hijo. Salud Pública de México, 51(6), 489-495. http://dx.doi.org/10.1590/S003636342009000600007

Genovesi, S., Giussani, M., Faini, A., Vigorita, F., Pieruzzi, F., Strepparava, M. G., et al. (2005). Maternal perception of excess weight in children: A survey conducted by paediatricians in the province of Milan. Acta Paediatrica, 94(6), 747-752. http:// dx.doi.org/10.1080/08035250510028894

Gómez, J. L. \& Canto, O. J. (1997). Percepción social. Psicología social. Madrid: Ediciones Pirámide.

Gupta, N., Goel, K., Shah, P. \& Misra, A. (2012). Childhood obesity in developing countries: Epidemiology, determinants, and prevention. Endocrine Reviews, 33(1), 48-70. http://dx.doi.org/10.1210/ er.2010-0028

Hirschler, V., Calcagno, M. L., Clemente, A. M., Aranda, C. \& González, C. (2008). Association between school children's overweight and maternal 
obesity and perception of their children's weight status. Journal of Pediatric Endocrinology $\mathbb{E}$ Metabolism, 21(7), 641-649. http://dx.doi.org/10.1515/ JPEM.2008.21.7.641

Huang, J. S., Becerra, K., Oda, T., Walker, E., Xu, R., Donohue, M., et al. (2007). Parental ability to discriminate the weight status of children: Results of a survey. Pediatrics, 120(1), e112-e119. http://dx.doi. org/10.1542/peds.2006-2143

Hughes, C. C., Sherman, S. N. \& Whitaker, R. C. (2010). How low-income mothers with overweight preschool children make sense of obesity. Qualitative Health Research, 20(4), 465-478. http://dx.doi. org/10.1177/1049732310361246

Jain, A., Sherman, S. N., Chamberlin, L. A., Carter, Y., Powers, S. W. \& Whitaker, R. C. (2001). Why don't low-income mothers worry about their preschoolers being overweight? Pediatrics, 107(5), 1138-1146. http://dx.doi.org/10.1542/peds.107.5.1138

Kuo, A. A., Inkelas, M., Lotstein, D. S., Samson, K. M., Schor, E. L. \& Halfon, N. (2006). Rethinking wellchild care in the United States: An international comparison. Pediatrics, 118(4), 1692-1702. http:// dx.doi.org/10.1542/peds.2006-0620

Lara-García, B., Flores-Peña, Y., Alatorre-Esquivel, M. A, Sosa-Briones, R. \& Cerda-Flores, R. M. (2011).
Percepción materna de sobrepeso-obesidad infantil y riesgos de salud en Nuevo Laredo, Tamaulipas, México. Salud Pública de México, 53(3), 258-263.

Tschamler, J. M., Conn, K. M., Cook, S. R. \& Halterman, J. S. (2010). Underestimation of children's weight status: Views of parents in an urban community. Clinical Pediatrics, 49(5), 470-476. Recuperado de http://dx.doi.org/10.1177/0009922809336071

Vanhala, M. L., Keinänen-Kiukaanniemi, S. M., Kaikkonen, K. M., Laitinen, J. H. \& Korpelainen, R. I. (2011, 24 de agosto). Factors associated with parental recognition of a child's overweight statusa cross sectional study. BMC Public Health, 11, 665. http://dx.doi.org/doi:10.1186/1471-2458-11-665

Wardle, J., Brodersen, N. H., Cole, T. J., Jarvis, M. J. \& Boniface, D. R. (2006). Development of adiposity in adolescence: Five year longitudinal study of an ethnically and socioeconomically diverse sample of young people in Britain. British Medical Journal, 332(7550), 1130-1135. http://dx.doi.org/10.1136/ bmj.38807.594792.AE

Warschburger, P. \& Kröller, K. (2009). Maternal perception of weight status and health risks associated with obesity in children. Pediatrics, 124(1), e60e68. http://dx.doi.org/10.1542/peds.2008-1845 